\title{
Biologic Therapies, Psoriasis, and COVID-19: Our Experience at the Psoriasis Unit of the University of Naples Federico II
}

\author{
Elisa Camela Gabriella Fabbrocini Eleonora Cinelli Wanda Lauro \\ Matteo Megna
}

Section of Dermatology, Department of Clinical Medicine and Surgery, University of Naples Federico II, Naples, Italy

Dear Editor,

Since the worldwide spread of SARS-CoV-2 infection, great concern arose on the safety of biologics, whose role has been extensively discussed on whether being beneficial, neutral, or detrimental in terms of susceptibility to the infection and/or severity of COVID-19 disease [1]. Biologics generally expose patients to an increased risk of contracting common and opportunistic infections; conversely, some classes of biologics, i.e. anti-interleukin (IL)-6 receptor, anti-IL-17A, and anti-tumor-necrosis factor (TNF)- $\alpha$, were demonstrated to limit the cytokine storm involved in the pathogenesis of COVID-19 disease by modulating specific cytokines [1].

Herein we report our experience regarding the biologic treatment of psoriasis patients during the COVID-19 pandemic at the University of Naples Federico II, Italy. During the pandemic (February 25, 2020, to June 25, 2020), 965 psoriasis patients (mean age 52.1 years, male $58.5 \%$ ) on biologics were interviewed about having been infected with SARS-CoV-2 virus, having had COVID-19 suspected symptoms (fever, dyspnea, cough, malaise), or having had contact with positive patients. Moreover, they were questioned about withdrawal or change in the due schedule of administration of biologics and, if so, whether the decision was voluntary or based on medical advice (Table 1). Of the 965 interviewed patients,
$311(32.2 \%)$ were under anti-TNF- $\alpha, 346$ (35.8\%) under IL-17, 64 (6.6\%) under anti-IL-23 therapy and 244 (25.0\%) on anti-IL-12/23.

Our analysis highlighted that $16 / 965$ patients $(1.6 \%)$ suspended the treatment during the pandemic: 3 (18.7\%) after seeking medical advice for fever and/or cough, while the remaining (81.2\%) were moved by the fear of being more susceptible to the infection. Likewise, 7 patients $(0.7 \%)$ delayed the administration of the biologic: 5 (71.4\%) voluntarily, while $2(28.6 \%)$ were instructed to suspend by the doctor after the occurrence of fever and/or cough.

Interestingly, only 1 patient $(0.1 \%)$, male and aged 74 years, with hypertension, contracted SARS-CoV-2 infection: although asymptomatic, he precautionarily suspended adalimumab for 1.5 months and restarted it after 2 negative swab results. He observed the house quarantine and did not require hospitalization or any related treatment.

Likewise, only a healthy 43-year-old woman (0.1\%) declared a strict contact with a SARS-CoV-2-positive patient but did not develop the infection. She precautionarily suspended etanercept and observed the quarantine, without hospitalization, then reintegrating the biologic.

Our data support that biologics may neither represent a risk factor for SARS-CoV-2 infection nor for a more severe disease. karger@karger.com

(c) 2020 S. Karger AG, Basel

www.karger.com/drm

Karger"
Matteo Megna

Section of Dermatology

Department of Clinical Medicine and Surgery, University of Naples Federico II

Via Pansini 5, IT-80131 Napoli (Italy)

mat24@libero.it 
Table 1. Main features of the study population $(n, \%)$ : patients' stratification according to biologic treatments and their suspension or delayed administration

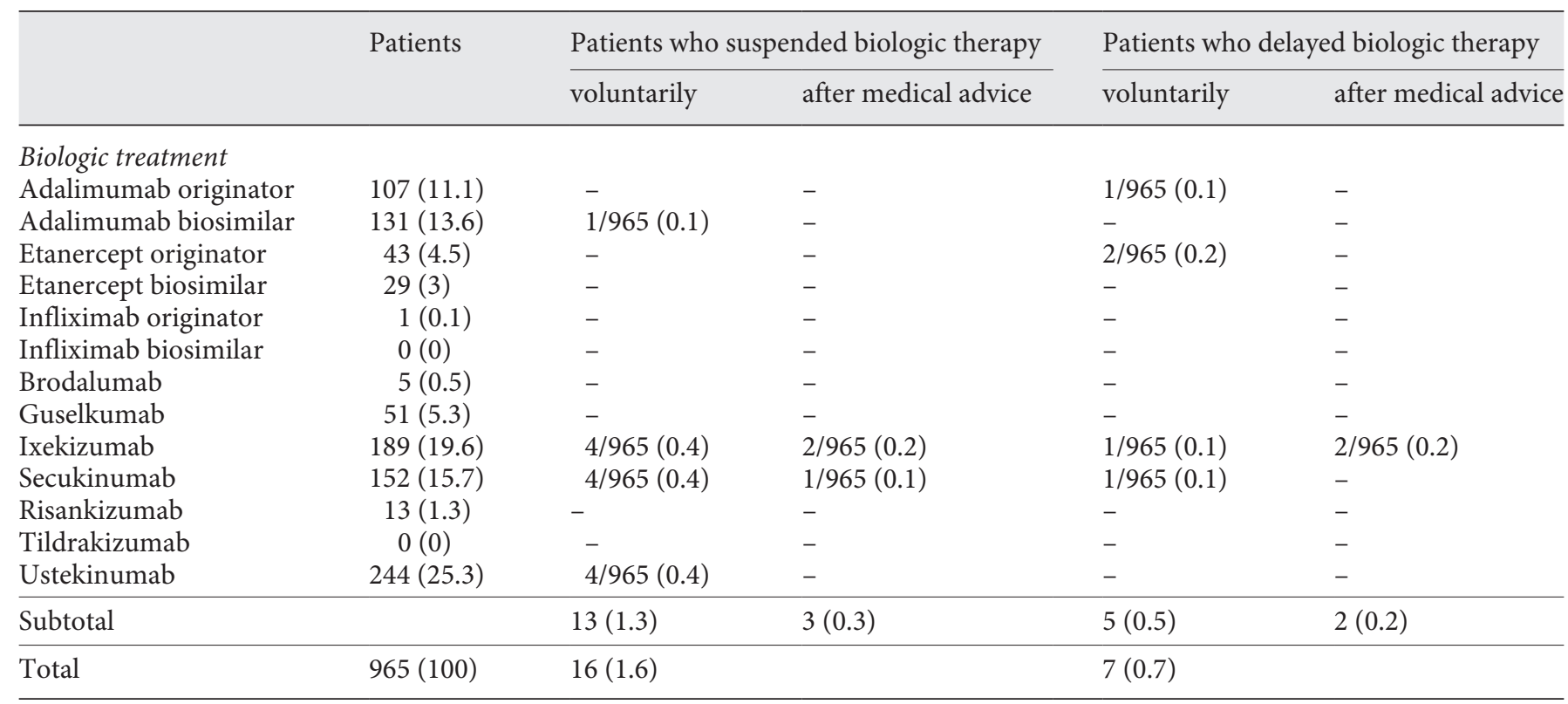

Such statements are in line with the most recent findings about the topic, as shown from the analysis run in two high-epidemic areas $(n=159$ patients at Bergamo Hospital and $n=139$ patients at Lecco Hospital, both treated with biologics), and so encourage adherence to biologics given the well-known effects at withdrawal such as flares and resistance to further treatments [2-5].

In this context, biologics for psoriasis seem to be an effective and safe therapy also during the COVID-19 pandemic. However, more research is needed to give consistency to our data and establish international guidelines on the management of psoriasis during the pandemic.

\section{Limitations}

Our study's limitations are the relatively short time of followup (4 months) and the lack of a control group (e.g., patients under conventional systemic treatments).

\section{Statement of Ethics}

The study was conducted ethically in accordance with the World Medical Association Declaration of Helsinki. Patients gave their written consent.

\section{Conflict of Interest Statement}

The authors have no conflicts of interest to declare.

\section{Funding Sources}

No funding is to declare.

\section{Author Contributions}

Elisa Camela: conception and design of the work. Gabriella Fabbrocini: acquisition, analysis, and interpretation of data for the work. Eleonora Cinelli: drafting the work for important intellectual content. Wanda Lauro: revising the work critically. Matteo Megna: final approval of the version to be published.

\section{References}

1 Amerio P, Prignano F, Giuliani F, Gualdi G. COVID-19 and psoriasis: should we fear for patients treated with biologics? Dermatol Ther (Heidelb). 2020 Jul;33(4):e13434

2 Nogueira M, Vender R, Torres T. Psoriasis, biologic therapy, and the pandemic of the 21st century. Drugs Context. 2020 May;9(May): 14.
3 Ebrahimi A, Sayad B, Rahimi Z. COVID-19 and psoriasis: biologic treatment and challenges. J Dermatolog Treat. Epub 2020 Jul 5.

4 Carugno A, Gambini DM, Raponi F, Vezzoli P, Locatelli AG, Di Mercurio M, et al. COVID-19 and biologics for psoriasis: a high-epidemic area experience - Bergamo, Lombardy, Italy. J Am Acad Dermatol. 2020 Jul;83(1):292-4.
5 Strippoli D, Barbagallo T, Prestinari F, Russo G, Fantini F. Biologic agents in psoriasis: our experience during coronavirus infection. Int $J$ Dermatol. 2020 Aug;59(8):e266-7. 